\title{
IDH2 compensates for IDH1 mutation to maintain cell survival under hypoxic conditions in IDH1-mutant tumor cells
}

\author{
YAO ZHANG ${ }^{1,2^{*}}$, WEIFENG LV ${ }^{1,2^{*}}$, QI LI ${ }^{1}$, QINHAO WANG ${ }^{2}$, YI RU ${ }^{2}$, \\ XIN XIONG ${ }^{2}$, FENGQI YAN ${ }^{2}$, TAO PAN $^{2}$, WEI LIN $^{1}$ and XIA LI ${ }^{2}$ \\ ${ }^{1}$ Department of Neurosurgery, Xijing Hospital; ${ }^{2}$ State Key Laboratory of Cancer Biology, \\ Department of Biochemistry and Molecular Biology, The Fourth Military Medical University, \\ Xi'an, Shaanxi 710032, P.R. China
}

Received December 17, 2018; Accepted May 1, 2019

DOI: $10.3892 / \mathrm{mmr} .2019 .10418$

\begin{abstract}
Mutations of isocitrate dehydrogenase (IDH) 1 and 2 occur in low-grade gliomas, acute myeloid leukemias and other types of solid cancer. By catalyzing the reversible conversion between isocitrate and $\alpha$-ketoglutarate $(\alpha-K G)$, IDH1 and 2 contribute to the central process of metabolism, including oxidative and reductive metabolism. IDH1 and 2 mutations result in the loss of normal catalytic function and acquire neomorphic activity, facilitating the conversion of $\alpha-\mathrm{KG}$ into an oncometabolite, (R)-2-hydroxyglutarate, which can cause epigenetic modifications and tumorigenesis. Small-molecule inhibitors of mutant IDH1 and 2 have been developed, and ongoing clinical trials have shown promising results in hematological malignancies, but not in gliomas. These previous findings make it necessary to identify the mechanism and develop more effective therapies for IDH1-mutant gliomas. In the present study, it was demonstrated that under hypoxic conditions, patient-derived primary glioma cells and HCT116 cells, both of which carry a monoallelic IDH1 arginine 132 to histidine mutation $(\mathrm{R} 132 \mathrm{H})$, have a slower growth rate than the corresponding wild-type IDH1 cells. Western blot analysis showed that IDH1 R132H-mutant cancer cells exhibited upregulated IDH2 protein expression under hypoxic conditions. Furthermore, the
\end{abstract}

Correspondence to: Dr Xia Li, State Key Laboratory of Cancer Biology, Department of Biochemistry and Molecular Biology, The Fourth Military Medical University, 169 Changle West Road, Xi'an, Shaanxi 710032, P.R. China

E-mail: lixia@fmmu.edu.cn

Dr Wei Lin, Department of Neurosurgery, Xijing Hospital, The Fourth Military Medical University, 127 Changle West Road, Xi'an, Shaanxi 710032, P.R.China

E-mail: linwei@fmmu.edu.cn

${ }^{*}$ Contributed equally

Key words: isocitrate dehydrogenase 1 arginine 132 histidine, isocitrate dehydrogenase 2, glioma, reductive carboxylation, small interfering RNA silencing of IDH2 using small interfering RNA significantly inhibited the growth of IDH1-mutant cells under hypoxic conditions. Finally, $\left[\mathrm{U}^{-13} \mathrm{C}_{5}\right.$ ] glutamine tracer analysis showed that IDH 2 knockdown reduced the reductive carboxylation of $\alpha-K G$ into isocitrate in HCT116 ${ }^{\mathrm{R} 132 \mathrm{H} /+}$ cells under hypoxic conditions. The present study showed for the first time, to the best of our knowledge, that IDH2 plays a compensatory role in maintaining reductive carboxylation-dependent lipogenesis and proliferation in IDH1 R132H tumor cells. Therefore, IDH2 could serve as a potential anti-tumor target for IDH1-mutant tumors, which may provide a new strategy for treatment.

\section{Introduction}

Mutations of isocitrate dehydrogenase (IDH) 1 and 2 have been reported to occur in most low-grade gliomas, acute myeloid leukemias and other solid tumors (1-2). IDH1 and 2 mutations disrupt the normal catalytic activity of the protein and they acquire a novel function, allowing them to reduce $\alpha$-ketoglutarate $(\alpha-K G)$ into $\mathrm{R}$-2-hydroxyglutarate (R-2HG) (1,3-6). Because R-2HG and $\alpha-\mathrm{KG}$ are structurally similar, 2-HG acts as a competitive inhibitor of the $\alpha-K G-d e p e n d e n t$ dioxygenases involved in epigenetic modifications, resulting in stem cell expansion and a cell differentiation block (4,6-9). Therefore, 2-HG functions as an oncometabolite to mediate tumorigenesis in cancer with mutant IDH1 and 2. Moreover, IDH1 and 2 mutations have been acknowledged as good biomarkers and potential drug targets (6-7).

There are three different isoforms of IDH; IDH1, 2 and 3. IDH1 is predominantly localized in the cytoplasm, while IDH2 and 3 are localized exclusively in the mitochondrial matrix. Although they catalyze a similar reaction, the oxidative decarboxylation of isocitrate into $\alpha-\mathrm{KG}$, they play different roles in cellular metabolism. IDH3, as a rate-limiting enzyme of the tricarboxylic acid (TCA) cycle, irreversibly catalyzes the NAD-dependent decarboxylation of isocitrate to generate $\alpha-\mathrm{KG}$. IDH1 and 2 are homodimeric enzymes that reversibly catalyze the oxidative decarboxylation of isocitrate to $\alpha-\mathrm{KG}$, as well as the reductive carboxylation of $\alpha-\mathrm{KG}$ to isocitrate. Therefore, IDH 1 and 2 play important roles in the regulation of the cellular redox status, glutamine metabolism and lipogenesis (10-13). 
Lipogenesis is an important metabolic process that provides a cellular energy source and structural components. Under normoxia, the precursor of fatty acid synthesis, acetyl coenzyme A (AcCoA), is predominantly generated from glucose-derived pyruvate via the glycolysis pathway. In mitochondria, the pyruvate dehydrogenase (PDH) complex mediates the conversion of pyruvate into AcCoA, which is then transferred into the cytosol and participates in lipid synthesis (14). However, under hypoxic conditions, the PDH complex is inhibited by hypoxia-inducible factor- $1 \alpha$ (HIF-1 $\alpha)$, resulting in the impaired generation of AcCoA from glucose (15). To support rapid proliferation, cells have to alter their metabolic pathways to accommodate the need for precursor AcCoA for fatty acid synthesis. Like glucose, glutamine has been recognized as another important source of carbon and nitrogen for biosynthetic reactions in mammalian cells. Glutamine can supply carbon to AcCoA through two major pathways. One pathway is described as glutaminolysis, wherein glutamine enters the TCA cycle as $\alpha-\mathrm{KG}$ and traverses in the forward direction to maintain oxidative phosphorylation $(13,16)$. The other pathway is called reductive carboxylation, in which glutamine enters the TCA cycle as $\alpha-\mathrm{KG}$ and is converted to isocitrate and citrate by reverse flux through the action of IDH1 and 2 (10-11,13). In an hypoxic microenvironment, tumor cells actively reprogram metabolism, and IDH1 and 2 play important roles in this process by mediating the reductive carboxylation of $\alpha-\mathrm{KG}$ into isocitrate, producing AcCoA to maintain lipid synthesis and cell survival (10,12-13).

IDH1 mutations, the most frequent of which is the arginine 132 to histidine mutation $(\mathrm{R} 132 \mathrm{H})$, inhibit not only the oxidative decarboxylation reaction but also the reductive carboxylation reaction $(3,5)$. Since tumor cells need to synthesize a large amount of lipids to support constant proliferation, IDH1 R132H was hypothesized to impair reductive carboxylation and lipid synthesis under hypoxic conditions, leading to reduced proliferation $(5,17)$. However, given that both IDH1 and 2 interchangeably support reductive carboxylation and lipid synthesis under hypoxic conditions $(5,18)$, the hypothesis that IDH2 might compensate for defective lipid synthesis and compromised cell proliferation in IDH1 R132H-mutant tumor cells requires investigation. To test this hypothesis, the present study determined the levels of IDH2 in IDH1 R132H-mutant patient-derived primary glioma cells and HCT116 cells, both of which harbor monoallelic IDH1 R132H, under normoxia and hypoxia. Furthermore, cell proliferation and reductive glutamine metabolism was examined in these cells with or without IDH2 knockdown. The results of the present study demonstrated that IDH2 compensates for IDH1 mutations to maintain reductive metabolism and the survival of IDH1-mutant cancer cells under hypoxic conditions.

\section{Materials and methods}

Primary glioma cells, HCT116 cells and glioma cell line U251 cell culture. Primary glioma cells were derived from fresh glioma tissue collected during brain tumor surgery from patients with grade-II astrocytomas; informed consent was obtained from the patients. Fresh tumor tissue was mechanically dissociated into $1 \mathrm{~mm}^{3}$ pieces in fresh DMEM (Gibco; Thermo Fisher Scientific, Inc.). The tumor fragments were placed at the bottom of culture flasks containing $10 \mathrm{ml}$ DMEM supplemented with penicillin, streptomycin and 10\% FBS (Gibco; Thermo Fisher Scientific, Inc.), and incubated at $37^{\circ} \mathrm{C}$ and $5 \% \mathrm{CO}_{2}$. The medium was replaced 3-5 days later to remove floating cells and tissues. The two individual patient-derived primary glioma cells, which were classified as grade-II astrocytomas, were confirmed to harbor either wild type (WT) IDH1 or monoallelic IDH1 R132H using genomic DNA sequencing (Sangon Biotech, Co., Ltd.) using the following primers: 5'-gcgtcaaatgtgccactatc-3' and 5'-ccttta gctaaatgtgtgta-3'. Experiments on these cells were performed within five passages.

Human colon cancer HCT116 ${ }^{\mathrm{IDH} 1 \mathrm{WT}}$ and HCT116 ${ }^{\mathrm{IDH} 1 \mathrm{R} 132 \mathrm{H} /+}$ cells, which harbor WT IDH1 and one allele of IDH1 R132H, respectively, were provided by Dr. Jing Ye (Department of Pathology, the Fourth Military Medical University) and cultured in McCoy's 5A modified medium (Gibco; Thermo Fisher Scientific, Inc.) with 10\% FBS. Human glioma cell lines U251 cells (Chinese Academy of Sciences Cell Bank), were cultured in DMEM (Gibco; Thermo Fisher Scientific, Inc.) supplemented with $10 \%$ FBS. For experiments under normoxia, cells were maintained in $21 \% \mathrm{O}_{2}, 74 \% \mathrm{~N}_{2}$ and $5 \% \mathrm{CO}_{2}$. For experiments under hypoxic conditions, cells were cultured under normoxia $\left(21 \% \mathrm{O}_{2}\right.$ at $\left.37^{\circ} \mathrm{C}\right)$ for $6 \mathrm{~h}$ to allow cells to adhere to the flask and were then moved to a hypoxic environment $\left(3 \% \mathrm{O}_{2}, 92 \% \mathrm{~N}_{2}\right.$ and $5 \% \mathrm{CO}_{2}$ at $\left.37^{\circ} \mathrm{C}\right)$.

Lentivirus packaging, cell infection and selection. The pLenti6, pLenti6-IDH1 and pLenti6-IDH1R132H vectors were generously gifted by Dr Jing Ye (Department of Pathology, The Fourth Military Medical University, Xi'an, China). For lentiviral packaging, 293T cells (Chinese Academy of Sciences Cell Bank) were seeded in $10 \mathrm{~cm}$ cell culture dishes and on reaching $\sim 60 \%$ confluence, the cells were transfected with the packaging system (7.5 $\mu \mathrm{g}$ psPAX2, $3.5 \mu \mathrm{g}$ pVSVG, $10 \mu \mathrm{g}$ pLenti plasmid) using Lipofectamine ${ }^{\circledR} 2000$ (Invitrogen; Thermo Fisher Scientific, Inc.), according to the manufacturer's protocol. Forty-eight hours later, the supernatant medium containing the virus was collected. The virus solution and fresh culture medium (DMEM supplemented with $10 \%$ FBS) were added to U251 cells in a 1:1 mixture when the cells reached $50-70 \%$ confluence. Twenty-four hours later, cells were selected with puromycin $(0.5 \mu \mathrm{g} / \mathrm{ml}$; Sigma-Aldrich; Merck KGaA) for two weeks to generate stable U251 cell lines expressing wild-type IDH1 or IDH1R132H.

Small interfering (si) RNAs and transfection. siRNAs that target two individual sites of the human IDH2 coding sequence were obtained from Shanghai GenePharma Co., Ltd. siIDH2-1, 5'-GCA AGA ACUAUGACGGAGA-3'; and siIDH2-2, 5'-CCCGUGUGGAAGAGUUCA ATT-3'. Nontargeting siRNA (5'-UUCUCCGAACGUGUCACGUTT-3'; Shanghai GenePharma Co., Ltd.) was used as a negative control. Cells were plated at a density of $1 \times 10^{5}$ into 6-well plates in DMEM without antibiotics. The cells were transfected $24 \mathrm{~h}$ after plating with $50 \mathrm{nM}$ siRNA targeting human IDH2 or nontargeting siRNA (scramble) as a negative control. Transfections were performed with Lipofectamine ${ }^{\circledR} 2000$, according to the manufacturer's protocol. Forty-eight hours following transfection, the knockdown efficiency was determined by western blot analysis. 
Proliferation assay. Cells were seeded in sextuplicate at a density of 2,000 cells/well in 96-well plates, and the medium was changed every 3 days. The number of viable cells/well at each time point was determined using a Cell Counting Kit-8 assay (CCK-8; Dojindo Molecular Technologies, Inc.), according to the manufacturer's protocol. Absorbance at $450 \mathrm{~nm}$ was measured $1 \mathrm{~h}$ after the addition of the CCK-8 solution.

Western blot analysis. Proteins were extracted using protein lysis buffer (Beyotime Institute of Biotechnology) in the presence of a protease inhibitor cocktail (Roche Diagnostics). The protein concentration was determined using the bicinchroninic acid Protein Assay kit (Thermo Fisher Scientific, Inc.). Equal amounts of protein samples, $20 \mu \mathrm{g} /$ lane, were separated by electrophoresis on $10 \%$ SDS-PAGE gels and were electrotransferred to nitrocellulose membranes. The membranes were blocked with $5 \%$ milk for $1.5 \mathrm{~h}$ at room temperature and then incubated with primary antibodies against IDH2 (1:500; cat. no. ab55271; Abcam), GAPDH (1:2,000; cat. no. 10494-1-AP; Wuhan Sanying Biotechnology) and HIF-1 $\alpha$ (1:1,000; cat. no. 14179; Cell Signaling Technology, Inc.) at $4^{\circ} \mathrm{C}$ overnight, followed by incubation with horseradish peroxidase-conjugated secondary antibodies (1:5,000; cat. nos. CW0102 and CW0103; Wuhan Sanying Biotechnology) for $1 \mathrm{~h}$ at room temperature. The signals were detected with an ECL substrate kit (Thermo Fisher Scientific, Inc.), and the immunoreactive bands were visualized using a gel-imaging analysis system (Tanon Science and Technology Co., Ltd.). The band intensity was quantified using ImageJ software (version 1.48; National Institutes of Health), wherein the relative values, using GAPDH as an internal control, of the first bands were designated as 1 .

Metabolic flux analysis by gas chromatography mass spectrometry $(G C-M S)$. HCT116 ${ }^{\mathrm{IDH} 1 \mathrm{R} 132 \mathrm{H} /+}$ cells, at a density of $1 \times 10^{6}$, were cultured in serum-reduced DMEM and transfected with $50 \mathrm{nM}$ IDH2-siRNA-1 or scramble using Lipofectamine ${ }^{\circledR} 2000$. After 48 h, the cells were cultured in medium containing $1 \mathrm{mM}$ uniformly ${ }^{13} \mathrm{C}$-labeled [U-13C] glutamine under hypoxic conditions for $24 \mathrm{~h}$, after which cells were collected for metabolic extraction. GC-MS analysis of metabolic flux was performed by Shanghai Biotech Co., Ltd., and the detailed procedure is described in the supplementary materials and methods.

Statistical analysis. Statistical analysis was performed using SPSS software (version 17; IBM Corp.). Each experiment was repeated independently a minimum of three times. Data are presented as the mean \pm SEM for multiple independent experiments or the mean \pm SD for technical replicates. Independent Student's t-test or one-way ANOVA followed by Fisher's least significant difference test were used to compare the continuous variables between two groups or among more than two groups, respectively. $\mathrm{P}<0.05$ was considered to indicate a statistically significant difference.

\section{Results}

Mutant IDHI cells grow more slowly than WT cells under hypoxic conditions. As IDH1 mutations reduce the ability of the enzyme to catalyze the bidirectional conversion between isocitrate and $\alpha-\mathrm{KG}$ (5), it was speculated that an IDH1 mutation would lead to compromised reductive metabolism and proliferation under hypoxic conditions. To test this hypothesis, the proliferation of IDH1 R132H-harboring patient-derived primary glioma cells was examined, which was previously established and confirmed using DNA sequencing (Fig. S1), H\&E staining and immunohistochemical staining (19). HCT116 cells that harbor one allele of IDH1 R132H (Fig. S2) were also used to test this hypothesis. Cells were cultured under normoxia or hypoxia for 3-5 days and subjected to a CCK-8 assay. Hypoxic conditions led to elevated HIF-1 $\alpha$ levels (Fig. S3). As shown in Fig. 1, under normoxia, IDH1-mutant primary glioma cells and $\mathrm{HCT} 116^{\mathrm{R} 132 \mathrm{H} /+}$ cells grew more slowly than WT IDH1 primary glioma cells and HCT116 ${ }^{\text {WT }}$ cells, respectively; however, these IDH1-mutant cells exhibited significantly repressed proliferation under hypoxic conditions compared with the proliferation of the WT counterpart. These results showed that IDH1 R132H cells are defective and exhibit reduced growth under hypoxic conditions.

R132H-mutant cancer cells upregulate IDH2 protein expression under hypoxic conditions. Both IDH1 and 2 are required to maintain the reverse TCA cycle for lipogenesis under hypoxic conditions $(10,13,18)$. As the growth of IDH1 R132H-mutant tumor cells was markedly inhibited under hypoxic conditions, it was hypothesized that these cells may compensate for the impaired lipid synthesis by overexpressing IDH2. To test this hypothesis, the expression of IDH2 in WT and IDH1 ${ }^{\mathrm{R} 132 \mathrm{H} /+}$ HCT116 cells and primary glioma cells was examined by western blot analysis. As shown in Fig. 2A and B, IDH2 was upregulated in both $\mathrm{HCT} 116^{\mathrm{R} 132 \mathrm{H} /+}$ and IDH1-mutant primary glioma cells relative to the expression in their WT counterparts under hypoxic conditions. Stable WT IDH1- and IDH1 R132H- overexpressing U251 cell lines, widely used as a gliobalstoma cell model (20), were established via lentivirus infection. Ectopic expression of IDH1 R132H consistently led to IDH2 upregulation under hypoxia compared with WT IDH1 (Fig. S4). These results suggested that IDH1-mutant cells may compensate for the adverse effects of an IDH1 mutation by upregulating IDH2 under hypoxic conditions.

Knockdown of IDH2 inhibits the growth of IDH1-mutant cells under hypoxic conditions. Although the IDH1-mutant cells were defective and grew more slowly under hypoxic conditions, these cells remained viable as shown in Fig. 1 (17). In the present study, it was also demonstrated that IDH1-mutant cells overexpressed IDH2 in hypoxic conditions (Fig. 2), suggesting that overexpression of IDH2 could compensate for the adverse effects caused by mutation of IDH1. Therefore, whether the knockdown of IDH2 would affect cell growth in these cells under hypoxic conditions was examined. For this purpose, two individual IDH2-siRNAs were transfected into WT HCT116 cells to determine their efficiency; the results showed that both siRNAs reduced IDH2 protein levels by $\sim 50 \%$ (Fig. 3A). WT and IDH $1^{132 \mathrm{H} /+}$ HCT116 cells and primary glioma cells were transfected with IDH2-siRNA or scramble siRNA, and then cultured under hypoxic conditions to examine proliferation. As shown in Fig. 3B and C, the inhibitory effect of IDH2 siRNAs on cell growth was comparable to that of the IDH1 $\mathrm{R} 132 \mathrm{H}$ mutation in both the HCT116 and primary glioma 
A HCT116

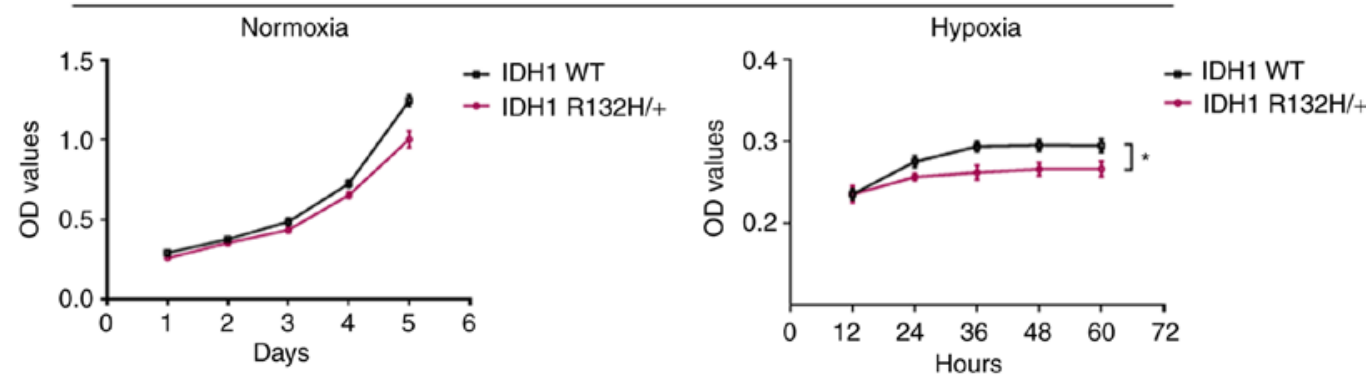

B

Primary glioma cells
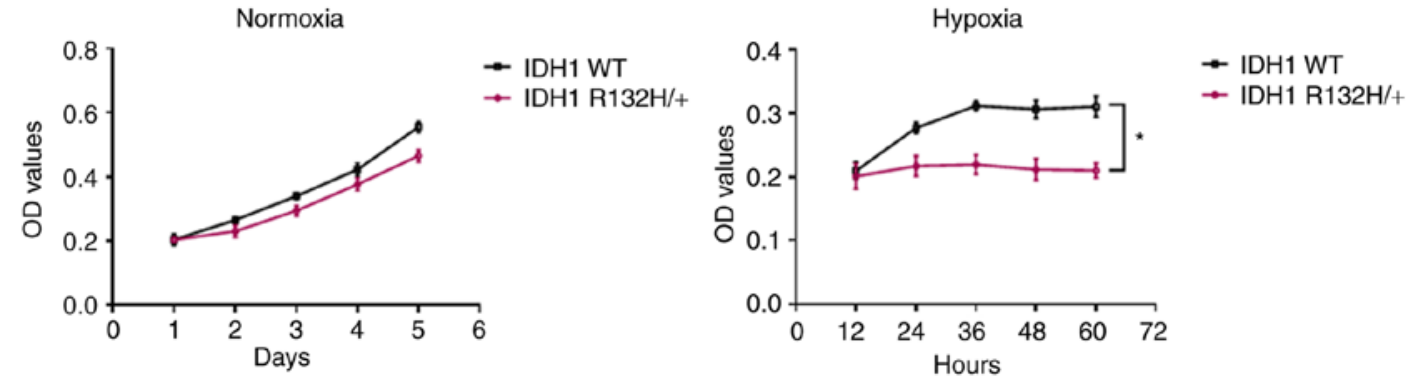

Figure 1. IDH1 R132H-mutant cells grow more poorly than WT cells under hypoxic conditions. (A) HCT116 and (B) patient-derived primary glioma cells harboring WT or a monoallelic IDHR132H mutation were cultured under normoxia or hypoxia for 3-5 days. Proliferation was measured using the Cell Counting Kit- 8 assay. The data are presented as the mean \pm SEM. ${ }^{*} \mathrm{P}<0.05$. IDH1, isocitrate dehydrogenase 1; WT, wild-type; OD, optical density; R132H, arginine 132 histidine.

cells. Moreover, IDH2 knockdown further slowed the growth of IDH1 R132H-mutant cells under hypoxic conditions. There data indicated that IDH2 knockdown exacerbated the growth defect in IDH1 R132H-mutant cells under hypoxic conditions.

Knockdown of IDH2 reduces the reductive carboxylation of IDH1-mutant cells in hypoxic conditions. As IDH1 and 2 are both involved in reductive carboxylation and lipogenesis under hypoxic conditions, it was reasoned that IDH2 knockdown may exacerbate the growth defect in hypoxic IDH1-mutant cells as a result of compromised reductive carboxylation. To detect the effect of IDH2 knockdown on reductive carboxylation, $\mathrm{IDH} 1^{\mathrm{R} 132 \mathrm{H} /+} \mathrm{HCT} 116$ cells were transfected with IDH2 siRNA1 or scramble siRNA and cultured in the presence of $\left[\mathrm{U}-{ }^{13} \mathrm{C}_{5}\right]$ glutamine under hypoxic conditions, and then collected for analysis of metabolic flux by GC-MS. The labeling metabolites derived from $\left[\mathrm{U}_{-}{ }^{13} \mathrm{C}_{5}\right]$ glutamine were different between oxidative and reductive metabolism; among the labeling metabolites, M5 citrate (Cit), M3 aspartate (Asp), M3 malate (Mal) and M3 fumarate (Fum) represented reductive metabolism, whereas M4 Asp, M4 Mal and M4 Fum represented oxidative metabolism (Fig. 4A). As shown in Fig. 4B, knockdown of IDH2 in IDH $1^{\mathrm{R} 132 \mathrm{H} /+} \mathrm{HCT} 116$ cells led to a significant decrease in the percentage of Cit (M5), Asp (M3), Mal (M3) and Fum (M3). These results suggested that IDH2 knockdown reduces reductive carboxylation under hypoxic conditions, thus impairing lipogenesis and cell growth in hypoxic IDH1-mutant cells.

\section{Discussion}

The discovery of IDH1 mutations has been an important breakthrough in the field, with the mechanism of IDH1 mutations and targeted therapy becoming popular research

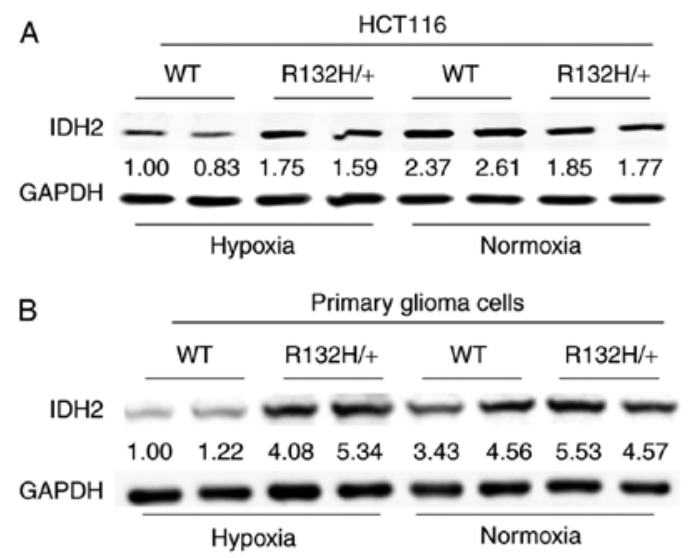

Figure 2. IDH1 R132H-mutant cells upregulate IDH2 protein expression under hypoxic conditions. (A) HCT116 and (B) patient-derived primary glioma cells harboring WT or monoallelic IDHR132H mutations were cultured under normoxia or hypoxia for $24 \mathrm{~h}$. IDH2 protein levels were determined by western blot analysis and quantified using Image J software. GAPDH was used as a loading control. The intensity of each band was quantified relative to the left-most IDH 2 band, which was designated as 1 . The values of the others bands are shown below the IDH2 bands. IDH1, isocitrate dehydrogenase 1; IDH2, isocitrate dehydrogenase 2; WT, wild-type; R132H, arginine 132 histidine; EV, empty vector.

topics $(2,7)$. Previous studies have established that $2-\mathrm{HG}$ is produced by mutant-IDH1 and causes profound epigenetic modifications and expansion of stem cells, and blocks differentiation (1,3-4,8-9,21). Small molecule mutant-IDH1 inhibitors have been developed and are at different stages of clinical trials; however, the currently available preclinical results have not been as promising as expected (2). It has been reported that targeting mutant IDH1 does not alter intracranial glioma growth in murine models (22). Although the blood-brain 
A
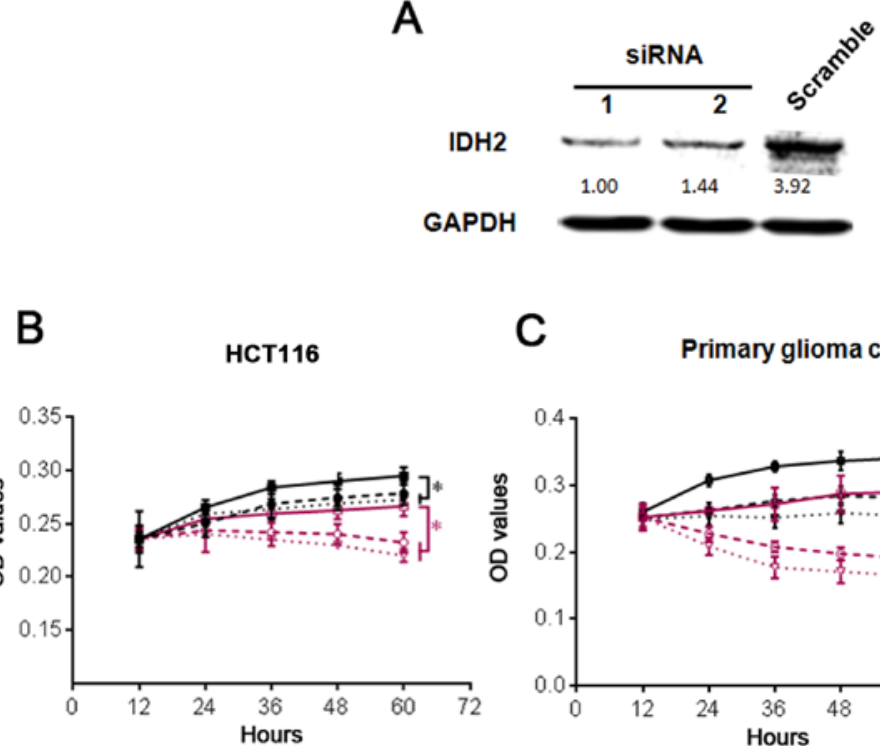

C Primary glioma cells

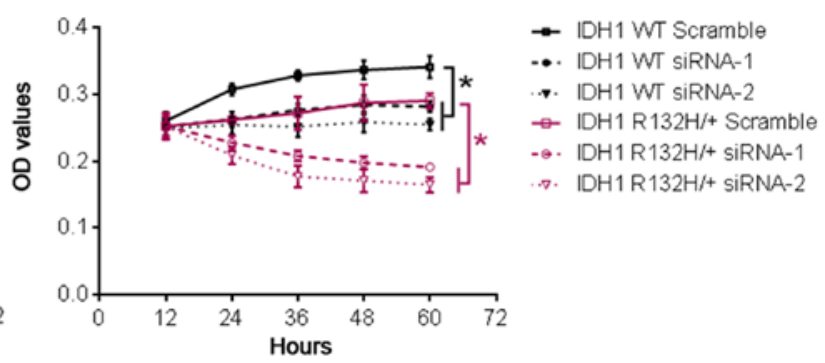

Figure 3. Knocking down IDH2 inhibits the growth of IDH1-mutant cells under hypoxic conditions. (A) WT HCT116 cells were transfected with scramble or two individual IDH2-targeting siRNAs. IDH2 protein levels were determined by western blot analysis, quantified using ImageJ software and shown below the bands (as described in Fig. 2), with GAPDH used as a loading control. (B) WT and IDH1 R132H-mutant HCT116 and (C) patient-derived primary glioma cells were transfected with two individual IDH2-siRNAs or scramble siRNA and cultured under hypoxic conditions for up to $60 \mathrm{~h}$. Proliferation was measured using the Cell Counting Kit-8 assay. The data are presented as the mean \pm SEM. "P<0.05. IDH1, isocitrate dehydrogenase 1; IDH2, isocitrate dehydrogenase 2; WT, wild-type; siRNA, small interfering RNA; R132H, arginine 132 histidine.

barrier or the irreversibility of the IDH mutation-induced epigenetic changes may account for the limited efficacy of IDH1 inhibitors (2), other alterations in cellular processes, including metabolism and signaling pathways, caused by mutant-IDH1, also play important roles in tumor progression. Therefore, it is important to understand the mechanism of mutant-IDH1 and to develop other therapeutic strategies. For the first time, to the best of our knowledge, the present study demonstrated that IDH1 R132H-mutant cells upregulate IDH2 as a compensatory mechanism to maintain reductive glutamine metabolism-dependent lipogenesis and cell proliferation under hypoxic conditions. As a result, the knockdown of IDH2 significantly inhibits the proliferation of IDH1 R132H-mutant cells under hypoxic conditions. Thus, IDH2 inhibition may serve as a new approach for the treatment of mutant-IDH1-harboring tumors.

Although mutations in IDH1 have been hypothesized to cause tumorigenesis, IDH1 is also a positive prognostic marker for patients with glioma (23). The finding that IDH1 $\mathrm{R} 132 \mathrm{H}$ inhibited the growth of glioma cell lines (24) and murine glioma progenitor cells (25) was proposed to result in a positive effect on patient survival (24). However, a previous study postulated that the survival benefit gained by IDH1 mutation is a result of sensitivity to temozolomide, rather than biological behavior (26). By contrast, Koivunen et al (27) reported that R-2HG produced by mutant-IDH1 enhanced the proliferation and soft agar growth of human astrocytes. A recent study showed that IDH1 R132H-knock-in in the mouse subventricular zone promoted the self-renewal and proliferation of neural stem cells (8). As such, the current opinions about mutant-IDH1 are contradictory, which may be due to the different cell types and contexts examined in previous studies.

In the present study, two IDH1 R132H-mutant cell models were used as models, including primary glioma cells and $\mathrm{HCT} 116^{\mathrm{R} 132 \mathrm{H} /+}$ cells, both of which harbor a single allelic IDH1 R132H mutation and consistently reflect the genetic IDH1 mutation status in patients. In the present study, IDH1-mutant cells were found to grow more slowly than their WT counterparts, particularly under hypoxic conditions. As mutations in IDH1 mitigate the oxidative and reductive activities of the enzyme $(3,5)$, it was concluded that the defective reductive glutamine metabolism caused by IDH1 mutation leads to reduced cell proliferation. This view is consistent with that of Grassian et al (17), who showed that cancer cells with a mutant IDH1 allele are unable to induce reductive glutamine metabolism under hypoxic conditions, thus compromising AcCoA and lipid production, leading to decreased cell growth. Therefore, it is proposed that the defective cell growth may account for the better prognosis of patients with IDH1 R132H mutation compared with those with WT-IDH1. Large-scale studies have established that IDH mutations together with other molecules are important markers for glioma classification and prognosis (6). A recent study, using genome-wide gene expression profiling, established a six-gene signature as an independent prognostic factor for IDH1-mutant glioma, and showed that the effect of IDH1 mutation is conserved across histological classifications; by combining histology grade, IDH1 status and the six-gene signature in all grades of glioma, patients could be stratified into six subgroups with distinct prognoses (28). Therefore, it would be interesting to observe the growth attributes of primary glioma cells derived from these different groups.

In the present study, data presented suggest that IDH2 was upregulated in both HCT116 IDH1 $1^{\mathrm{R} 132 \mathrm{H} /+}$ cells and IDH1-mutant primary glioma cells under hypoxic conditions. This finding suggests that cancer cells can compensate for the adverse effects caused by mutation of IDH1 by 

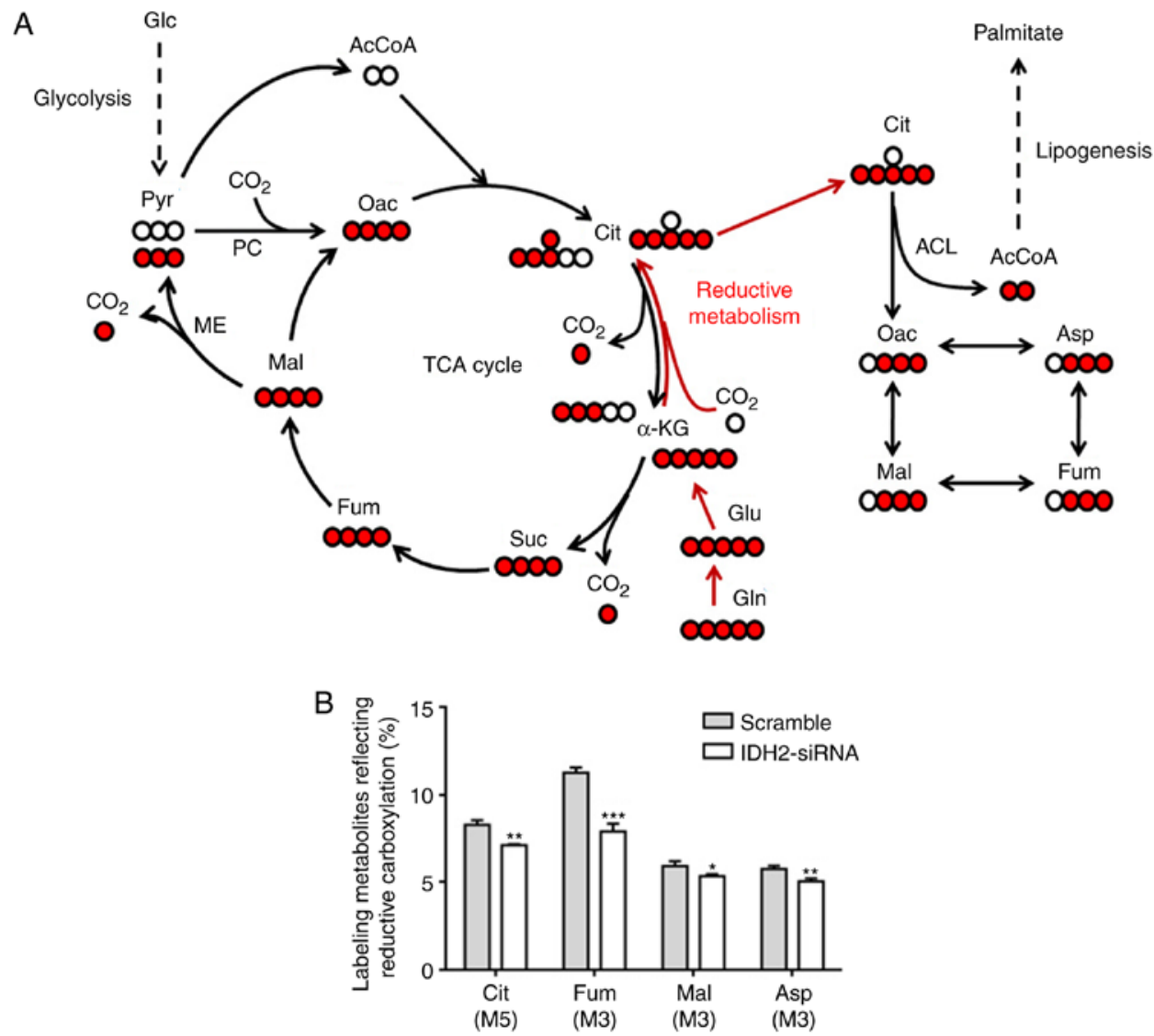

Figure 4. Knockdown of IDH2 reduces the reductive carboxylation of IDH1-mutant cells in hypoxic conditions. (A) A schematic diagram of carbon atom (circles) transitions, showing labeling metabolites derived from $\left[\mathrm{U}_{-}{ }^{13} \mathrm{C}_{5}\right]$ glutamine through oxidative (black arrows) and reductive (red arrows) pathways. Isotopic-labeled metabolites generated through the reductive carboxylation pathway include M5 Cit, M3 Asp, M3 Mal and M3 Fum. Red circles represent ${ }^{13} \mathrm{C}$ carbon atoms, white circles represent non- ${ }^{13} \mathrm{C}$ marked carbon atoms. (B) HCT116 ${ }^{\text {IDHIR132H/+ }}$ cells were transfected with IDH2 siRNA1 or scramble siRNA and cultured in the presence of $\left[\mathrm{U}_{-}^{13} \mathrm{C}_{5}\right]$ glutamine for $24 \mathrm{~h}$ under hypoxic conditions. The cells were then collected for gas chromatography-mass spectrometry analysis of the metabolites. The relative abundance of reductive carboxylation-specific mass isotopomers are shown as the mean $\pm \mathrm{SD}(\mathrm{n}=3)$. ${ }^{*} \mathrm{P}<0.05$, ${ }^{* *} \mathrm{P}<0.01$, ${ }_{* * * *} \mathrm{P}<0.005$. IDH2, isocitrate dehydrogenase 2; siRNA, small interfering RNA; TCA, tricarboxylic acid; Glc, glucose; Pyr, pyruvate; AcCoA, acetyl co-enzyme A; Oac, oxaloacetate; Cit, citrate; Mal, malate; Asp, aspartate; Fum, fumarate; $\alpha$-KG, $\alpha$-ketoglutarate; Suc, succinate; Gln, glutamine; Glu, glutamate; PC, pyruvate carboxylase; ME, malic enzyme; ACL, ATP citrate lyase.

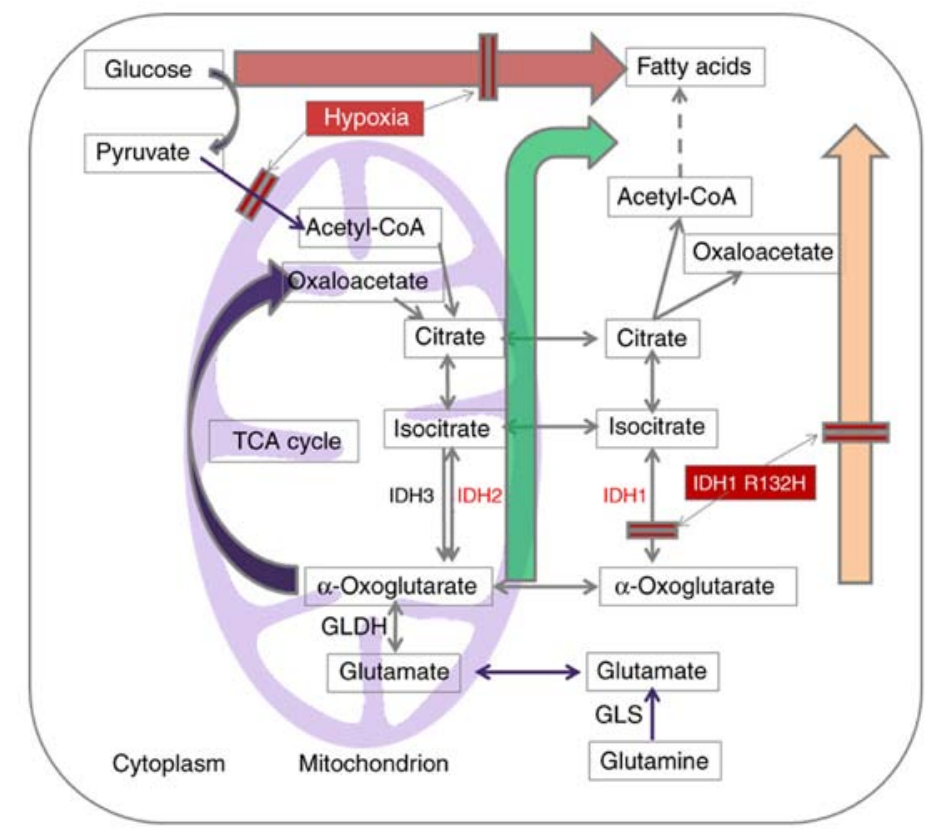

Figure 5. A model depicting the metabolic reprograming of IDH1 R132H-mutant cells under hypoxic conditions. IDH2 compensates for IDH1R132H in reductive glutamine metabolism, permitting lipogenesis and proliferation to continue. IDH1, isocitrate dehydrogenase 1; IDH2, isocitrate dehydrogenase 2; IDH3, isocitrate dehydrogenase 3; CoA, co-enzyme A; TCA, tricarboxylic acid; GLDH, glutamate dehydrogenase; GLS, glutaminase. 
upregulating IDH2 under hypoxic conditions. This allows processes, including reductive carboxylation and lipogenesis, that are required for cell proliferation, to continue. This is consistent with the findings of Mustafa et al (29), who examined the genes participating in the TCA cycle and anaerobic glycolysis in 33 IDH1 mutated and 39 IDH1 WT glioma samples and found that the expression levels of several genes were different, among which IDH2 was reported to be upregulated. However, Chen et al (25) reported no change in IDH2 expression in the expression profiling data of 46 IDH1 $\mathrm{R} 132 \mathrm{H}$ high-grade gliomas compared with 163 WT-IDH1 high-grade gliomas. Such a discrepancy may result from the different datasets analyzed or the different oxygen concentrations the glioma samples were exposed to. The findings of the present study showed that IDH2 was upregulated in IDH1-mutant cells under hypoxic conditions; therefore, IDH2 may serve as a therapeutic target for the treatment of IDH1-mutant cancer. Consistent with this proposed treatment strategy, the present study found that targeting IDH2 with siRNAs significantly decreased reductive carboxylation and inhibited the proliferation of cancer cells with a monoallelic IDH1 mutation.

A limitation of the present study is the use of a limited sample number and tumor grading, a WT and mutant grade-II astrocytoma. However, similar results were obtained with WT and IDH1 R132H/+ HCT116 cells. IDH2 upregulation was also observed in the stable U251 glioblastoma cell line, which overexpresses IDH1 R132H, but not WT IDH1, under hypoxic conditions. Furthermore, it was proposed in a genome-wide gene expression profiling study that the effect of mutations in IDH1 is conserved across histological classifications (28). Therefore, the compensatory role played by IDH2 could apply to a wider range of cancer cells containing mutations in IDH1. Previous studies have shown that mutation of IDH1 disrupts the reductive activity used to generate citrate for lipid synthesis (5), and also increases glutaminolysis to form lipids $(6,25,30)$, indicating the presence and importance of compensatory mechanisms. Glioma cells with mutant IDH1 were found to be sensitive to the inhibition of glutaminase or glutamate dehydrogenase $(25,31)$. Therefore, the results of the present study together with data presented in the literature provide potential and promising therapeutic strategies that exploit the compensatory mechanisms caused by the mutation of IDH1. Further systematic studies, including in vivo examination of the effects of siRNAs targeting the compensatory molecules, are required in the future. In addition, future studies should investigate whether IDH1 can compensate for mutations of IDH2 in IDH2 mutant malignancies.

In conclusion, the present study reports, for the first time, to the best of our knowledge, that IDH2 compensates for the IDH1 R132H mutation to maintain cell survival under hypoxic conditions in IDH1-mutant tumor cells. Thus, IDH2 may serve as a potential antitumor target for IDH1 mutant tumors.

\section{Acknowledgements}

The authors thank Dr Jing Ye and Mr. Chao Wang (The Fourth Military Medical University) for their kind assistance with the IDH1 $1^{\mathrm{R} 132 \mathrm{H} /+} \mathrm{HCT} 116$ cells and other experimental materials used in this present study.

\section{Funding}

This study was supported by grants from the National Natural Science Foundation of China (grant nos. 81572504 and 81572469), the Natural Science Foundation of Shaanxi Province (grant no. 2016JZ028), and the Science and Technology Reseach and Development Program of Shaanxi Province (grant no. 2016SF-094).

\section{Availability of data and materials}

The materials used in the current study are available from the corresponding author on reasonable request.

\section{Authors' contributions}

YZ, WLv and QL performed the primary glioma cell culture and cellular experiments. QW and YR conducted the statistical analysis. FY, TP and XX performed routine cell culture transfections. WLi and XL designed the study and wrote the manuscript. All authors have read and approved the final manuscript.

\section{Ethics approval and consent to participate}

The present study was approved by the Ethics Committee of Xijing Hospital, the Fourth Military Medical University (approval no. 20180122). All human subjects recruited for this study provided written informed consent prior to participation.

\section{Patient consent for publication}

All human subjects whose samples were used for this study provided written informed consent for publication.

\section{Competing interests}

The authors declare that they have no competing interests.

\section{References}

1. Yan H, Parsons DW, Jin G, McLendon R, Rasheed BA, Yuan W, Kos I, Batinic-Haberle I, Jones S, Riggins GJ, et al: IDH1 and IDH2 mutations in gliomas. N Engl J Med 360: 765-773, 2009.

2. Waitkus MS, Diplas BH and Yan H: Biological role and therapeutic potential of IDH mutations in cancer. Cancer Cell 34: 186-195, 2018.

3. Dang L, White DW, Gross S, Bennett BD, Bittinger MA, Driggers EM, Fantin VR, Jang HG, Jin S, Keenan MC, et al: Cancer-associated IDH1 mutations produce 2-hydroxyglutarate. Nature 462: 739-744, 2009.

4. Zhao S, Lin Y, Xu W, Jiang W, Zha Z, Wang P, Yu W, Li Z, Gong L, Peng Y, et al: Glioma-derived mutations in IDH1 dominantly inhibit IDH1 catalytic activity and induce HIF-1alpha. Science 324: 261-265, 2009.

5. Leonardi R, Subramanian C, Jackowski S and Rock CO: Cancer-associated isocitrate dehydrogenase mutations inactivate NADPH-dependent reductive carboxylation. J Biol Chem 287: 14615-14620, 2012.

6. Waitkus MS, Diplas BH and Yan H: Isocitrate dehydrogenase mutations in gliomas. Neuro Oncol 18: 16-26, 2016.

7. Yang H, Ye D, Guan KL and Xiong Y: IDH1 and IDH2 mutations in tumorigenesis: Mechanistic insights and clinical perspectives. Clin Cancer Res 18: 5562-5571, 2012. 
8. Bardella C, Al-Dalahmah O, Krell D, Brazauskas P, Al-Qahtani K, Tomkova M, Adam J, Serres S, Lockstone H, Freeman-Mills L, et al: Expression of Idh1 $1^{\mathrm{R} 132 \mathrm{H}}$ in the murine subventricular zone stem cell niche recapitulates features of early gliomagenesis. Cancer Cell 30: 578-594, 2016.

9. Lu C, Ward PS, Kapoor GS, Rohle D, Turcan S, Abdel-Wahab O, Edwards CR, Khanin R, Figueroa ME, Melnick A, et al: IDH mutation impairs histone demethylation and results in a block to cell differentiation. Nature 483: 474-478, 2012.

10. Filipp FV, Scott DA, Ronai ZA, Osterman AL and Smith JW: Reverse TCA cycle flux through isocitrate dehydrogenases 1 and 2 is required for lipogenesis in hypoxic melanoma cells. Pigment Cell Melanoma Res 25: 375-383, 2012.

11. Koh HJ, Lee SM, Son BG, Lee SH, Ryoo ZY, Chang KT, Park JW, Park DC, Song BJ, Veech RL, et al: Cytosolic NADP+-dependent isocitrate dehydrogenase plays a key role in lipid metabolism. J Biol Chem 279: 39968-39974, 2004.

12. Wise DR, Ward PS, Shay JE, Cross JR, Gruber JJ, Sachdeva UM, Platt JM, DeMatteo RG, Simon MC and Thompson CB: Hypoxia promotes isocitrate dehydrogenase-dependent carboxylation of $\alpha$-ketoglutarate to citrate to support cell growth and viability. Proc Natl Acad Sci USA 108: 19611-19616, 2011.

13. Metallo CM, Gameiro PA, Bell EL, Mattaini KR, Yang J, Hiller K, Jewell CM, Johnson ZR, Irvine DJ, Guarente L, et al: Reductive glutamine metabolism by IDH1 mediates lipogenesis under hypoxia. Nature 481: 380-384, 2011.

14. Hatzivassiliou G, Zhao F, Bauer DE, Andreadis C, Shaw AN, Dhanak D, Hingorani SR, Tuveson DA and Thompson CB: ATP citrate lyase inhibition can suppress tumor cell growth. Cancer Cell 8: 311-321, 2005

15. Kim JW, Tchernyshyov I, Semenza GL and Dang CV: HIF-1-mediated expression of pyruvate dehydrogenase kinase: A metabolic switch required for cellular adaptation to hypoxia. Cel Metab 3: 177-185, 2006.

16. Wise DR, DeBerardinis RJ, Mancuso A, Sayed N, Zhang XY, Pfeiffer HK, Nissim I, Daikhin E, Yudkoff M, McMahon SB and Thompson CB: Myc regulates a transcriptional program that stimulates mitochondrial glutaminolysis and leads to glutamine addiction. Proc Natl Acad Sci USA 105: 18782-18787, 2008.

17. Grassian AR, Parker SJ, Davidson SM, Divakaruni AS, Green CR, Zhang X, Slocum KL, Pu M, Lin F, Vickers C, et al: IDH1 mutations alter citric acid cycle metabolism and increase dependence on oxidative mitochondrial metabolism. Cancer Res 74: 3317-3331, 2014.

18. Mullen AR, Wheaton WW, Jin ES, Chen PH, Sullivan LB, Cheng T, Yang Y, Linehan WM, Chandel NS and DeBerardinis RJ: Reductive carboxylation supports growth in tumour cells with defective mitochondria. Nature 481: 385-388, 2011.

19. Zhang Y, Wei X, Wang Q, Zhang M, L X, Lin W: Culture of primary human astrocytoma cell with isocitrate dehydrogenase (IDH1) R132H mutation. J Modern Oncol 25: 843-847, 2017 (In Chinese).
20. Fujikawa A, Sugawara H, Tanaka T, Matsumoto M, Kuboyama K, Suzuki R, Tanga N, Ogata A, Masumura M and Noda M: Targeting PTPRZ inhibits stem cell-like properties and tumorigenicity in glioblastoma cells. Sci Rep 7: 5609, 2017.

21. Turcan S, Rohle D, Goenka A, Walsh LA, Fang F, Yilmaz E, Campos C, Fabius AW, Lu C, Ward PS, et al: IDH1 mutation is sufficient to establish the glioma hypermethylator phenotype. Nature 483: 479-483, 2012.

22. Pusch S, Krausert S, Fischer V, Balss J, Ott M, Schrimpf D, Capper D, Sahm F, Eisel J, Beck AC, et al: Pan-mutant IDH1 inhibitor BAY 1436032 for effective treatment of IDH1 mutant astrocytoma in vivo. Acta Neuropathol 133: 629-644, 2017.

23. Leu S, von Felten S, Frank S, Vassella E, Vajtai I, Taylor E, Schulz M, Hutter G, Hench J, Schucht P, et al: IDH/MGMT-driven molecular classification of low-grade glioma is a strong predictor for long-term survival. Neuro Oncol 15: 469-479, 2013.

24. Bralten LB, Kloosterhof NK, Balvers R, Sacchetti A, Lapre L, Lamfers M, Leenstra S, de Jonge H, Kros JM, Jansen EE, et al: IDH1 R132H decreases proliferation of glioma cell lines in vitro and in vivo. Ann Neurol 69: 455-463, 2011.

25. Chen R, Nishimura MC, Kharbanda S, Peale F, Deng Y,Daemen A, Forrest WF, Kwong M, Hedehus M, Hatzivassiliou G, et al: Hominoid-specific enzyme GLUD2 promotes growth of IDH1R132H glioma. Proc Natl Acad Sci USA 111: 14217-14222, 2014.

26. Houillier C, Wang X, Kaloshi G, Mokhtari K, Guillevin R, Laffaire J, Paris S, Boisselier B, Idbaih A, Laigle-Donadey F, et al: IDH1 or IDH2 mutations predict longer survival and response to temozolomide in low-grade gliomas. Neurology 75: 1560-1566, 2010.

27. Koivunen P, Lee S, Duncan CG, Lopez G, Lu G, Ramkissoon S, Losman JA, Joensuu $\mathrm{P}$, Bergmann $\mathrm{U}$, Gross $\mathrm{S}$, et al: Transformation by the (R)-enantiomer of 2-hydroxyglutarate linked to EGLN activation. Nature 483: 484-488, 2012.

28. Cheng W, Ren X, Zhang C, Cai J, Han S and Wu A: Gene expression profiling stratifies IDH1-mutant glioma with distinct prognoses. Mol Neurobiol 54: 5996-6005, 2017.

29. Mustafa DA, Swagemakers SM, Buise L, van der Spek PJ and Kros JM: Metabolic alterations due to IDH1 mutation in glioma: Opening for therapeutic opportunities? Acta Neuropathol Commun 2: 6,2014.

30. Reitman ZJ, Duncan CG, Poteet E, Winters A, Yan LJ, Gooden DM, Spasojevic I, Boros LG, Yang SH and Yan H: Cancer-associated isocitrate dehydrogenase 1 (IDH1) $\mathrm{R} 132 \mathrm{H}$ mutation and d-2-hydroxyglutarate stimulate glutamine metabolism under hypoxia. J Biol Chem 289: 23318-23328, 2014.

31. Seltzer MJ, Bennett BD, Joshi AD, Gao P, Thomas AG, Ferraris DV, Tsukamoto T, Rojas CJ, Slusher BS, Rabinowitz JD, et al: Inhibition of glutaminase preferentially slows growth of glioma cells with mutant IDH1. Cancer Res 70: 8981-8987, 2010. 